\title{
Transient Heat Conduction
}

\author{
Jed Brody \\ Department of Physics \\ Emory University
}

\section{Purpose:}

To measure and analyze the "cold front" passing through a material dipped in ice water.

\section{Theory: heat conduction}

Consider a rectangular solid at room temperature. Place its bottom surface in contact with ice water. Heat will flow from the solid into the water. For simplicity, neglect heat flow from air into the solid. (This approximation is based on the fact that air conducts heat less effectively than water.) Under this assumption, the temperature will not vary in the horizontal directions. The temperature will only vary in the vertical direction, $\mathrm{z}$. So we want to determine $T(z, t)$, the temperature of every point within the solid at all times. $\mathrm{T}(\mathrm{z}, \mathrm{t})$ satisfies the heat conduction equation:

$$
\frac{\partial T}{\partial t}=D \frac{\partial^{2} T}{\partial z^{2}},
$$

where $\mathrm{D}$ is called the thermal diffusivity of the solid. (Some authors use $\alpha$ instead of D.)

This equation looks a little complicated, but we can understand it qualitatively. Suppose that some horizontal layer in the solid is hotter than the neighboring layers above and below. This would appear as a local maximum in the graph of T vs. z. So $\partial^{2} T / \partial z^{2}$ is negative (curves are concave down at local maxima), and so $\partial \mathrm{T} / \partial \mathrm{t}$ is also negative, according to Equation (1). This makes sense: if Layer $\mathrm{A}$ is hotter than the neighboring layers, then heat will flow out of Layer A into its neighbors. Consequently, Layer A's temperature will decrease.

In order to solve Equation (1), we need some more information. Specifically, we need boundary conditions (what is happening at the bottom and top of the solid?) and an initial condition (what is the temperature distribution in the solid before we place it in contact with ice water?).

We will use the following boundary conditions:

$$
\begin{aligned}
& \mathrm{T}(0, \mathrm{t})=0^{\circ} \mathrm{C} \\
& \partial \mathrm{T}(\mathrm{H}, \mathrm{t}) / \partial \mathrm{z}=0 \\
& \mathrm{~T}(\mathrm{z}, 0)=\mathrm{T}_{\mathrm{a}}
\end{aligned}
$$


Equation (2) says that the temperature of the bottom of the solid $(\mathrm{z}=0)$ is $0^{\circ} \mathrm{C}$, the temperature of the ice water. Equation (3) says that no heat flows through the top of the solid; this is the condition for thermal insulation. Equation (4) says that the initial temperature of the entire solid is $\mathrm{T}_{\mathrm{a}}$, the ambient temperature.

To solve Equation (1), we use separation of variables: $T(z, t)=Z(z) \tau(t)$. Plugging this into Equation (1) yields $\mathrm{Z}(\mathrm{d} \tau / \mathrm{dt})=\tau \mathrm{D}\left(\mathrm{d}^{2} \mathrm{Z} / \mathrm{dz}^{2}\right)$. Let's put all the $\mathrm{t}$ dependence on one side, and all the $z$ dependence on the other: $(1 / Z)\left(d^{2} Z / d z^{2}\right)=(1 / D \tau)(d \tau / d t)$. This is true for all $\mathrm{z}$ and all $\mathrm{t}$, which is only possible if both sides of the equation are equal to the same constant, $-\mathrm{k}^{2}$. (For convenience, we choose $-\mathrm{k}^{2}$ instead of just $\mathrm{k}$.) So we have $\mathrm{d} \tau / \mathrm{dt}$ $=-\mathrm{Dk}^{2} \tau$ and $\mathrm{d}^{2} \mathrm{Z} / \mathrm{dz}^{2}=-\mathrm{k}^{2} \mathrm{Z}$. So $\tau=\exp \left(-\mathrm{k}^{2} \mathrm{Dt}\right)$ and $\mathrm{Z}=\mathrm{A} \sin (\mathrm{kz})+\mathrm{B} \cos (\mathrm{kz})$, where $\mathrm{A}$ and $\mathrm{B}$ are unknown constants. So one solution is $\mathrm{T}(\mathrm{z}, \mathrm{t})=$ $[A \sin (k z)+B \cos (k z)] \exp \left(-k^{2} \mathrm{Dt}\right)$. The lower boundary condition, Equation (2), allows us to infer that $B$ is 0 , so $T(z, t)=A \sin (k z) \exp \left(-k^{2} D t\right)$.

So what's k? Equation (3) allows us to write $\cos (\mathrm{kH})=0$, which means $\mathrm{kH}=\pi / 2+\mathrm{p} \pi$ for any whole number $p$. So $k=(\pi / H)(p+1 / 2)$. Since there are many possible values of $\mathrm{k}$, we need to sum all the possible solutions:

$$
T(z, t)=\sum_{p=0}^{\infty} A_{p} \sin \left[\left(p+\frac{1}{2}\right) \pi z / H\right] \exp \left[-\left(p+\frac{1}{2}\right)^{2} \pi^{2} D t / H^{2}\right]
$$

Finally, we need to determine the values $A_{p}$. To do this, we use the initial condition, Equation (4):

$$
T(z, 0)=\sum_{p=0}^{\infty} A_{p} \sin \left[\left(p+\frac{1}{2}\right) \pi z / H\right]=T_{a} .
$$

If we multiply this by $\sin [(\mathrm{q}+1 / 2) \pi \mathrm{z} / \mathrm{H}]$ and integrate from 0 to $\mathrm{H}$, all the terms in the sum will integrate to 0 except the one with $\mathrm{p}=\mathrm{q}$. So

$$
\begin{aligned}
& \int_{0}^{H} A_{p} \sin ^{2}\left[\left(p+\frac{1}{2}\right) \pi z / H\right] d z=\int_{0}^{H} T_{a} \sin \left[\left(p+\frac{1}{2}\right) \pi z / H\right] d z \\
& \frac{A_{p} H}{2}=\frac{H T_{a}}{\left(p+\frac{1}{2}\right) \pi} \\
& A_{p}=\frac{2 T_{a}}{\left(p+\frac{1}{2}\right) \pi}
\end{aligned}
$$

So Equation (5) becomes 


$$
T(z, t)=\sum_{p=0}^{\infty} \frac{2 T_{a}}{\left(p+\frac{1}{2}\right) \pi} \sin \left[\left(p+\frac{1}{2}\right) \pi z / H\right] \exp \left[-\left(p+\frac{1}{2}\right)^{2} \pi^{2} D t / H^{2}\right],
$$

which is our final solution. Wow!

Since $\mathrm{T}$ depends on $\mathrm{z}$, there is a thermal gradient. A thermal gradient creates a refractive gradient because the index of refraction depends on the temperature. When we shine light through the material, the light will be bent by this refractive gradient. The bending is proportional to $\partial \mathrm{T} / \partial \mathrm{z}$, so let's calculate this from Equation (7).

$$
\frac{\partial T}{\partial z}=\sum_{p=0}^{\infty} \frac{2 T_{a}}{H} \cos \left[\left(p+\frac{1}{2}\right) \pi z / H\right] \exp \left[-\left(p+\frac{1}{2}\right)^{2} \pi^{2} D t / H^{2}\right] .
$$

By shining light through the solid and measuring the bending of the beam, we can determine $\partial \mathrm{T} / \partial \mathrm{z}$ as a function of time for whichever $\mathrm{z}$ we've chosen. Figure 1 shows Equation (8) as a function of time for a particular value of $\mathrm{z}$. You will see that your measurements of beam bending will closely follow the shape of this curve.

Neglecting convection, the preceding analysis applies to liquids in rectangular containers.

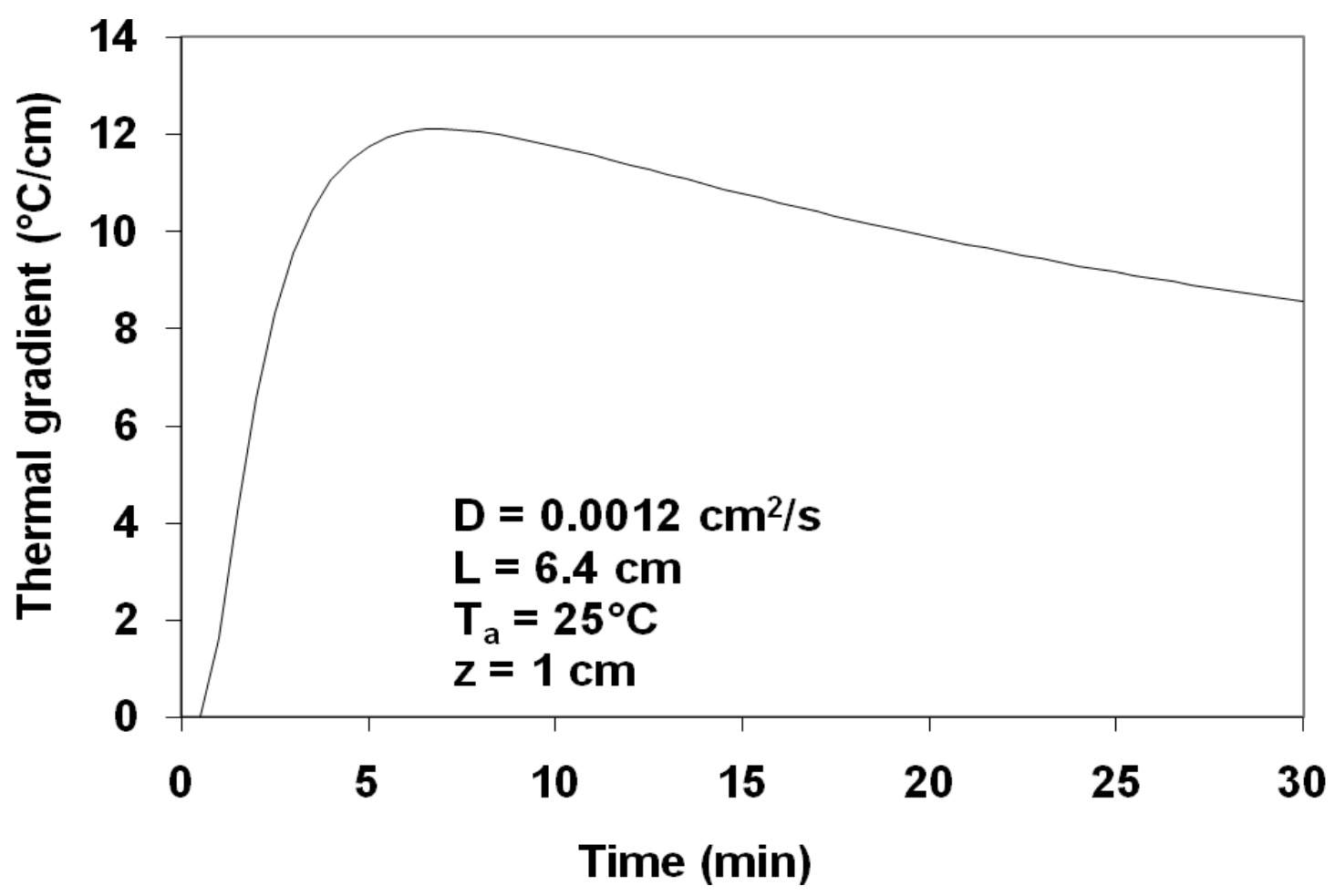

Figure 1. Thermal gradient as a function of time after placing the bottom of the solid in contact with ice water. 
You might wonder why there's a little kink in the curve around 1 min. The reason is that there is an infinite number of terms in Equation (8), but I only use the first 21 terms. The higher order terms (higher $\mathrm{p}$ ) are extremely small at high $\mathrm{t}$ : each term decays exponentially as $\exp \left[-(\mathrm{p}+1 / 2)^{2} \pi^{2} \mathrm{Dt} / \mathrm{H}^{2}\right]$. However, as $\mathrm{t}$ approaches 0 , all the infinite terms approach the same magnitude as the first term, and using only 21 terms causes noticeable error at small t.

\section{Theory: Refractive gradients}

Figure 2 illustrates the experimental situation. A laser illuminates the side of a rectangular material.

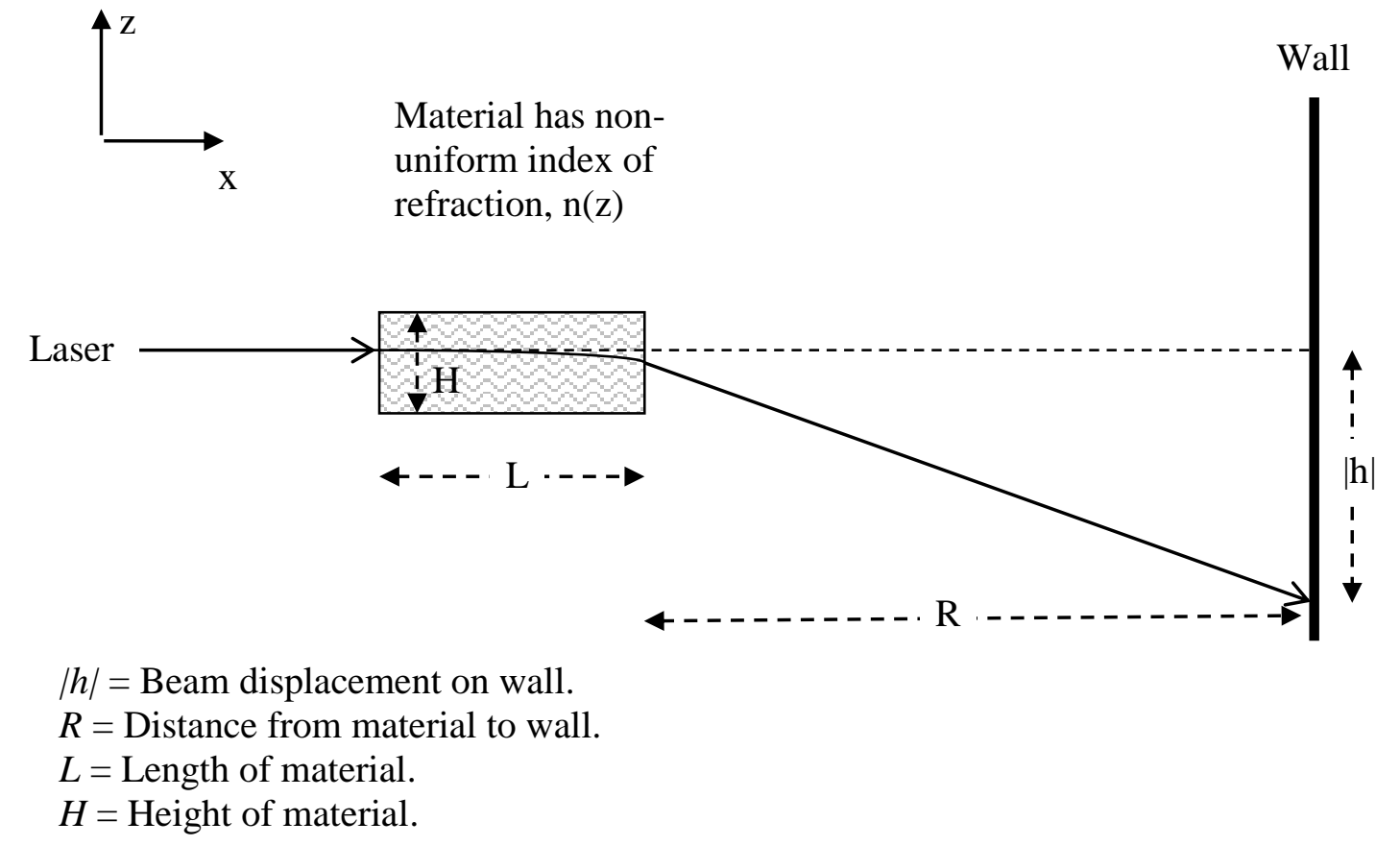

Figure 2. An illustration defining some experimental parameters.

We will show that the beam displacement $\mathrm{h}$ is proportional to the refractive gradient in the material:

$$
\mathrm{h}=(\mathrm{dn} / \mathrm{dz}) \mathrm{RL}
$$

Equation (9) says that we can determine the refractive gradient (dn/dz) simply by shining light through the medium and measuring the beam displacement on the wall. While deriving Equation (9), we will also derive the amazing fact that light follows a parabolic path through a medium whose refractive index varies linearly with height. First, let's write an expression for the refractive index, $n(z)$ :

$$
\mathrm{n}(\mathrm{z})=\mathrm{n}_{0}+\nabla \mathrm{n} \cdot \mathrm{z}
$$


$\nabla \mathrm{n}$ is defined as dn/dz; let's assume that $\nabla \mathrm{n}$ is constant. Then, Equation (10) just says that $\mathrm{n}(\mathrm{z})$ varies linearly with $\mathrm{z}$. We will also assume that $\nabla \mathrm{n}$ is small, so that $\mathrm{n}(\mathrm{z})$ is always nearly $\mathrm{n}_{0}$.

How can we apply Snell's Law to a material with variable $n$ ? We must divide the material up into an infinite number of differential slices, each with constant $n$. Let's consider just the first few differential slices. We'll apply Snell's Law at the interface between each pair of adjacent slices.

Let's imagine "zooming in" on the material where the laser enters. The refractive index $\mathrm{n}$ varies continuously with $\mathrm{z}$, but we divide the material into differential slices of constant n. This is shown in Figure 3.

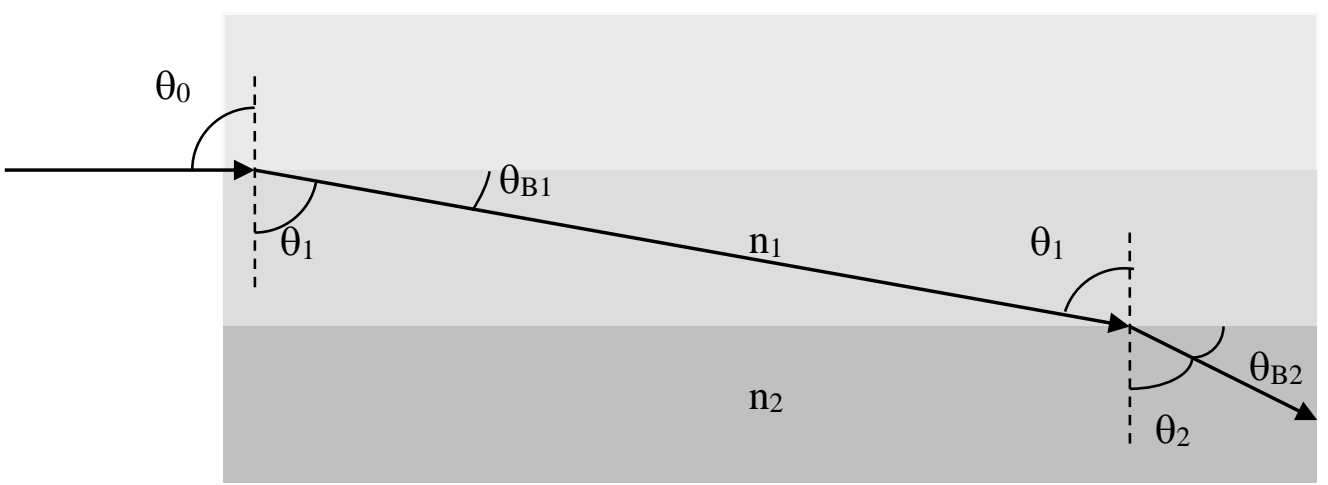

Figure 3. A "close-up" of the small region of the material where the laser enters.

Consider the leftmost ray of light. It enters the material "skimming" the interface between the $\mathrm{n}_{0}$ slice and the $\mathrm{n}_{1}$ slice. Let's imagine that the light continues undeflected for an infinitesimal distance. Now we can apply Snell's Law at the interface between $\mathrm{n}_{0}$ and $\mathrm{n}_{1}$. We first draw the normal to the interface (the dotted line). Then we observe that the angle of incidence, $\theta_{0}$, is $90^{\circ}$ since we choose to shine the laser normal to the side of the material. We want to find the angle of refraction, $\theta_{1}$, and then its complement, $\theta_{\mathrm{B} 1}$.

$$
\begin{array}{ll}
\mathrm{n}_{0} \sin \theta_{0}=\mathrm{n}_{1} \sin \theta_{1} & \text { from Snell's Law } \\
\mathrm{n}_{0}=\mathrm{n}_{1} \sin \theta_{1} & \text { from } \theta_{0}=90^{\circ} \\
\mathrm{n}_{0}=\mathrm{n}_{1} \cos \theta_{\mathrm{B} 1} & \text { from } \theta_{1}+\theta_{\mathrm{B} 1}=90^{\circ}
\end{array}
$$

You might object that $90^{\circ}$ is an unreasonable angle of incidence. However, $90^{\circ}$ can be an angle of refraction in total internal reflection. So Equation (11.b) is like total internal reflection in reverse. 
Notice that Equation (11.b) implies $\mathrm{n}_{0}<\mathrm{n}_{1}$. This means that $\mathrm{n}$ is increasing downward. If $\mathrm{n}$ were increasing upward, the beam would bend upward.

Now let's consider the ray when it strikes the interface between the $n_{1}$ slice and the $n_{2}$ slice.

$$
\begin{array}{ll}
\mathrm{n}_{1} \sin \theta_{1}=\mathrm{n}_{2} \sin \theta_{2} & \text { from Snell's Law } \\
\mathrm{n}_{1} \cos \theta_{\mathrm{B} 1}=\mathrm{n}_{2} \cos \theta_{\mathrm{B} 2} & \text { from } \theta_{1}+\theta_{\mathrm{B} 1}=90^{\circ} \text { and } \theta_{2}+\theta_{\mathrm{B} 2}=90^{\circ} \\
\mathrm{n}_{0}=\mathrm{n}_{1} \cos \theta_{\mathrm{B} 1}=\mathrm{n}_{2} \cos \theta_{\mathrm{B} 2} & \text { from }(11 . \mathrm{b}) \text { and }(12 . \mathrm{b})
\end{array}
$$

Equation (12.c) has an amazing implication: if we continued applying Snell's Law at additional interfaces (between the $n_{2}$ slice and the $n_{3}$ slice, between the $n_{3}$ slice and the $n_{4}$ slice, etc.), we would find $n_{0}=n_{3} \cos \theta_{B} 3, n_{0}=n_{4} \cos \theta_{B}$, etc. So $n_{0}$ always equals the product of $n$ and $\cos \theta_{\mathrm{B}}$. We can write this conclusion as

$$
\mathrm{n}_{0}=\mathrm{n} \cos \theta_{\mathrm{B}}
$$

where both $\mathrm{n}$ and $\theta_{\mathrm{B}}$ are functions of $\mathrm{z}$. Here we have stopped thinking about discrete, differential slices (which were just a conceptual tool) and returned to thinking of $\mathrm{n}$ as a function of a continuous variable. Equation (13) says that if we know $\mathrm{n}_{0}$ and $\mathrm{n}$ at any point, we can determine $\theta_{\mathrm{B}}$, the "bending angle" (the angle to the horizontal), at that point.

Let's assume that $\theta_{\mathrm{B}}$ is small, so that

$$
\cos \left[\theta_{\mathrm{B}}(\mathrm{z})\right] \approx 1-\theta_{\mathrm{B}}^{2} / 2
$$

Now we can substitute Equations (10) and (14) into Equation (13):

$$
\mathrm{n}_{0}=\left(\mathrm{n}_{0}+\nabla \mathrm{n} \cdot \mathrm{z}\right)\left(1-\theta_{\mathrm{B}}^{2} / 2\right)
$$

Each binomial on the right side of Equation (15) contains a large term and a small correction term. We will neglect the product of the two small terms. Then,

$$
\mathrm{n}_{0}=\mathrm{n}_{0}+\nabla \mathrm{n} \cdot \mathrm{z}-\mathrm{n}_{0} \theta_{\mathrm{B}}^{2} / 2
$$

which becomes

$$
\mathrm{n}_{0} \theta_{\mathrm{B}}{ }^{2} / 2=\nabla \mathrm{n} \cdot \mathrm{z}
$$

or

$$
\theta_{B}=\sqrt{\frac{2 \nabla n \cdot z}{n_{0}}} .
$$


Now let's see how $\theta_{\mathrm{B}}$ is related to the tangent to the light path. $\theta_{\mathrm{B}}$ is the angle between the horizontal and the tangent to the light path. In Figure 4 , we see that $\theta_{\mathrm{B}} \approx \tan \theta_{\mathrm{B}}=$ $\mathrm{dz} / \mathrm{dx}$.

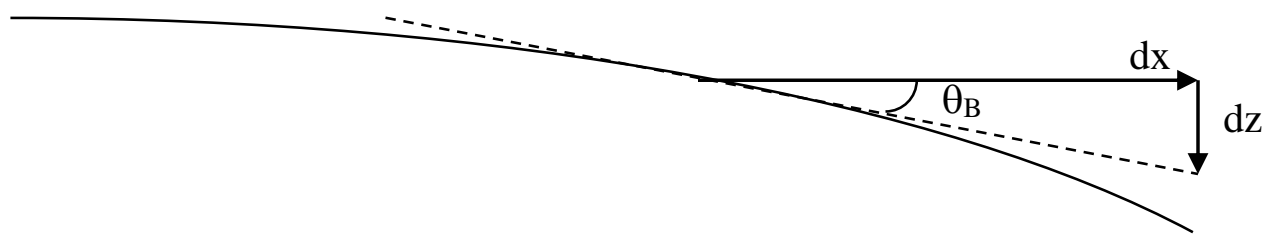

Figure 4. Relationship between $\theta_{\mathrm{B}}$ and the tangent to the light path.

Substituting $\theta_{\mathrm{B}}=\mathrm{dz} / \mathrm{dx}$ into Equation (18),

$$
\frac{d z}{d x}=\sqrt{\frac{2 \nabla n \cdot z}{n_{0}}},
$$

or

$$
\frac{d z}{\sqrt{z}}=\sqrt{\frac{2 \nabla n}{n_{0}}} d x .
$$

Upon integration,

$$
2 \sqrt{z}=x \sqrt{\frac{2 \nabla n}{n_{0}}},
$$

or

$$
z=\frac{\nabla n}{2 n_{0}} x^{2} .
$$

So the light takes a parabolic path (downward, because $\nabla n=d n / d z$ is negative: $n$ decreases at higher $\mathrm{z}$ ). If we extended Figure 3 and drew a large number of sequential, infinitesimal segments of the light's path, a parabola would form. Finally, let's look at where the light leaves the material, as shown in Figure 5: 


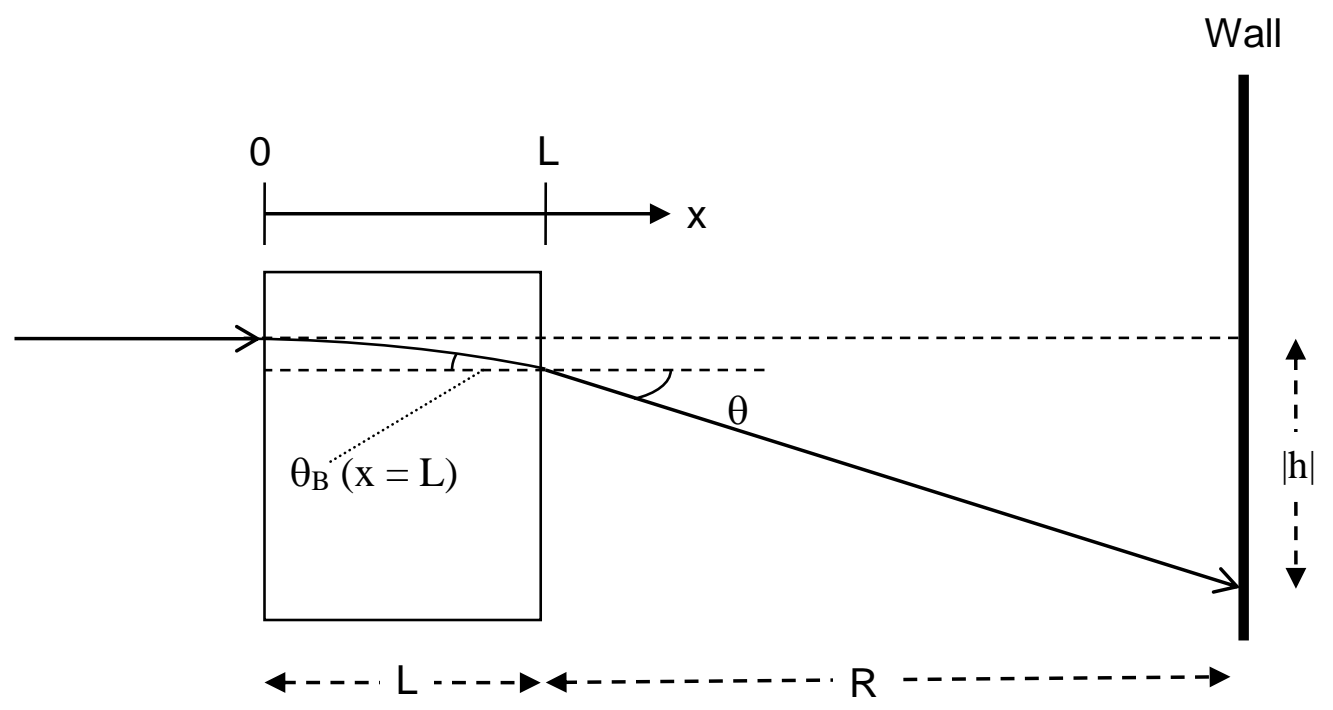

Figure 5. The angles used in Snell's Law where the light leaves the material.

Snell's Law where the light leaves the material says that

$$
\mathrm{n}(\mathrm{z}) \cdot \sin \theta_{\mathrm{B}}=1 \cdot \sin \theta
$$

where 1 is the index in air. The index $n(z)$ is approximately $n_{0}$ because the variation in $n$ is slight. Moreover, both $\theta_{\mathrm{B}}$ and $\theta$ are small, so we can rewrite Equation (23) as

$$
\mathrm{n}_{0} \theta_{\mathrm{B}}=\theta \text {. }
$$

Applying $\theta_{\mathrm{B}}=\mathrm{dz} / \mathrm{dx}$ to Equation (24) yields $\theta_{\mathrm{B}}=\mathrm{x} \nabla \mathrm{n} / \mathrm{n}_{0}$, but since $\mathrm{x}=\mathrm{L}$ where the light leaves the material, $\theta_{\mathrm{B}}=\mathrm{L} \nabla \mathrm{n} / \mathrm{n}_{0}$. From the geometry of Figure $5, \theta \approx \mid \mathrm{h} / \mathrm{R}$. Thus Equation (24) becomes

$$
\mathrm{n}_{0}\left(\mathrm{~L} \nabla \mathrm{n} / \mathrm{n}_{0}\right)=|\mathrm{h}| / \mathrm{R}
$$

which at last simplifies to Equation (9).

Since $d n / d z=(d n / d T)(\partial T / \partial z)$, we can rewrite Equation $(9)$ as

$$
\mathrm{h}=(\mathrm{dn} / \mathrm{dT})(\partial \mathrm{T} / \partial \mathrm{z}) \mathrm{RL}
$$

where $\partial \mathrm{T} / \partial \mathrm{z}$ is given by Equation (8). If we measure R, L, H, and $\mathrm{z}$, and $\mathrm{h}$ as a function of time, we can fit our data to Equation (26) by using dn/dT and D as fitting parameters. 


\section{4. $\underline{\text { Instructions }}$}

\section{EXPERIMENT}

- This experiment can be performed with any transparent material. You can try Plexiglass, glycerol, and water.

- To record $\mathrm{h}$ as a function of time, you can use a digital camera to make a movie of the beam's changing position on the wall. Alternatively, you can use the Neanderthal method of directly marking the wall with the beam's position every thirty seconds or so.

- Make sure you record $\mathrm{H}, \mathrm{R}, \mathrm{L}$, and $\mathrm{z}$, in addition to the time-varying $\mathrm{h}$ !

- It might make sense to do a few preliminary measurements to understand the experiment. Then, develop a computer model of Equations (7) and (8), as described below. Based on your analysis, design experiments that will show you a wide range of behaviors. (In other words, what values of $\mathrm{z}$ and $\mathrm{H}$ should you use? In each case, how long should you record data?)

\section{ANALYSIS}

- One important goal is to fit your data to Equation (26). The interesting term in this equation is $\partial \mathrm{T} / \partial \mathrm{z}$, given by Equation (8). I used Excel to create Figure 1. Excel works fine, but programming languages like Python and MATLAB are more versatile, and I can help you with it if you want to practice programming.

- To fit Equation (26) to your data, you can use D and dn/dT as fitting parameters. $\mathrm{D}$ affects the shape of the curve, and dn/dT affects only the height. If necessary, you might try adjusting $\mathrm{z}$ a little in case your measurement was insufficiently accurate.

- After achieving a reasonable fit, you can compare D and dn/dT to published values.

- Strive for the deepest possible understanding of how the "cold front" moves through the material. To achieve this, you can generate some theoretical curves of Equations (7) and (8). For example, you might show T vs. $\mathrm{z}$ at various times on the same graph.

- Ultimately, the data and theoretical curves mean nothing without a strong conceptual understanding. Explain qualitatively why your measurements and simulations make sense. Specifically, why does the beam bend down and then come back up again?

\section{Acknowledgement}

This experiment was suggested by Phil Segre. 